\title{
Capsule Commentary on Zullig et al., Primary Care Providers' Acceptance of Pharmacists' Recommendations to Support Optimal Medication Management for Patients with Diabetic Kidney Disease
}

\author{
Tony Kuo, M.D., M.S.H.S. \\ Health, Los Angeles, CA, USA. \\ J Gen Intern Med 35(1):406 \\ DOI: $10.1007 / \mathrm{s} 11606-019-05498-2$ \\ (c) Society of General Internal Medicine 2019
}

UCLA Clinical and Translational Science Institute and Division of Chronic Disease and Injury Prevention, Los Angeles County Department of Public

$\mathrm{Z}$ ullig et al. ${ }^{1}$ conducted an observational analysis of a subset of participants randomized to the intervention arm of the STOP-DKD clinical trial. The intent of the study was to describe whether primary care physicians' (PCPs') acceptance of pharmacists' recommendations impacted systolic blood pressure (SBP) at 36 months from baseline. Participants in the intervention received monthly telephone calls with a pharmacist addressing health behaviors and medication management. The research pharmacists made recommendations to PCPs to aid in titration decisions. In all, they made 176 treatment recommendations for 59 participants, of which $61 \%$ were accepted by the PCPs. While there was a significant association between the number of recommendations and the odds of PCP acceptance, there was no association between accepted recommendations and SBP.

A number of factors may have led to the mixed results. First, hypertension management in diabetics is different than in patients with essential hypertension. ${ }^{2}$ Second, medication adherence can be influenced by multiple factors and interactions at the patient-, provider-, and healthcare-system level. ${ }^{3}$ This complexity may have reduced the possibility of showing a robust impact in a relatively small sample size. Third, the structure of the management team seemed somewhat complicated-i.e., each patient was essentially interacting with two providers (pharmacist and $\mathrm{PCP}$ ), with the former making the phone calls and recommendations, with the latter making the decisions but without continuity in patient contact. More recent models of medication management utilize the pharmacist as the primary provider who directly titrates a patient's medications based on a protocol. This type of comprehensive medication management $(\mathrm{CMM})$ is gradually being recognized as a key practice for team-based care. ${ }^{4}$

Despite these shortcomings, Zullig et al.'s research contributes to an emerging body of evidence that supports the use of CMM or its variations (e.g., this study) in various settings. Emerging

Published online November 1, 2019 data also suggest that these interventions could be cost-effective, and in some instances, revenue generating. The integration of CMM into a patient-centered medical home in South Carolina, for example, resulted in the practice being able to collect $\sim \$ 7400$ / month for the pharmacist services and a $20.6 \%$ increase in the average daily payments for physician services. ${ }^{5}$

Corresponding Author: Tony Kuo, M.D., M.S.H.S.; UCLA Clinical and Translational Science Institute and Division of Chronic Disease and Injury Prevention, Los Angeles County Department of Public Health, Los Angeles, CA, USA (e-mail: tkuo@mednet.ucla.edu).

Compliance with Ethical Standards:

Conflict of Interest: The author declares that he does not have a conflict of interest.

Publisher's Note: Springer Nature remains neutral with regard to jurisdictional claims in published maps and institutional affiliations.

\section{REFERENCES}

1. Zullig LL, Jazowski SA, Davenport CA, Diamantidis CJ, Oakes MM, Patel S, Moaddeb J, Bosworth HB. Primary care providers' acceptance of pharmacists' recommendations to support optimal medication management for patients with diabetic kidney disease. J Gen Intern Med. https://doi.org/ 10.1007/s11606-019-05403-x.

2. Passarella P, Kiseleva TA, Valeeva FV, Gosmanov AR. Hypertension management in diabetes: 2018 update. Diab Spectrum. 2018;31(3):218224. doi: https://doi.org/10.2337/ds17-0085.

3. McBane SE, Dopp AL, Abe A, Benavides S, Chester EA, Dixon DI, Dunn M, Johnson MD, Nigro SJ, Rothrock-Christian T, Schwartz AH, Thrasher K, Walker S. ACCP White Paper: Collaborative drug therapy management and comprehensive medication management-2015. Pharmacotherapy. 2015;35(4):e39-e50. doi: https://doi.org/10.1002/phar. 1563.

4. Butler A, Dehner M, Gates RJ, Shane P, Chu M, DeMartini L, Nunez de Ybarra J, Peck C, McInnis T, Chen S. Proposed model. Comprehensive medication management programs: 2015 status in Southern California. Res Soc Admin Pharm. 2017;13:63-87. doi: https://doi.org/10.1016/j. sapharm.2016.02.003.

5. Favel PH, Wagner T, Ziegler B, Fleming PA, Davis RE. Payment models. A sustainable business model for comprehensive medication management in a patient-centered medical home. J Am Pharm Assoc. 2019;59:285-290. doi: https://doi.org/10.1016/j.japh.2018.11.001.

Publisher's Note Springer Nature remains neutral with regard to jurisdictional claims in published maps and institutional affiliations. 